\title{
COMPARATIVE STUDY OF GELATINASE ACTIVITY AND PELLICLE FORMATION AMONG EXTENDED-SPECTRUM BETA-LACTAMASE AND NON-EXTENDED-SPECTRUM BETA-LACTAMASE PRODUCING ACINETOBACTER BAUMANNII FROM DIABETIC FOOT ULCER INFECTIONS
}

\author{
DIWAN MAHMOOD KHAN ${ }^{1,2}$, I. VENKATAKRISHNA RAO ${ }^{2}$, M. S. MOOSABBA ${ }^{1 *}$ \\ ${ }^{1}$ Department of General Surgery, Yenepoya Medical College Hospital, Yenepoya (Deemed to be University), Mangalore, Karnataka, India. \\ ${ }^{2}$ Department of Microbiology, Yenepoya Medical College Hospital, Yenepoya (Deemed to be University), Mangalore, Karnataka, India. \\ Email: drmssonkal@yahoo.in
}

Received: 10 July 2018, Revised and Accepted: 09 August 2018

ABSTRACT

Objective: The aim of the study was to assess and compare the gelatinase activity and pellicle formation in extended-spectrum beta-lactamase (ESBL) and non-ESBL producing Acinetobacter baumannii isolates from diabetic foot ulcer infection (DFI).

Methods: A total of 42 isolates of A. baumannii recovered from patients of DFI from September 2016 to February 2018 . Isolates were identified by the standard microbiological method and confirmed by the BD Phoenix 100 system. The antimicrobial susceptibility test was performed by the Kirby-Bauer disk diffusion method and ESBL was detected by double disk diffusion synergy test method. Gelatinase production was determined by the Luria Bertani agar supplemented with $30 \mathrm{~g} / \mathrm{L}$ gelatin, and pellicle formation was determined by the Mueller-Hinton broth which is incubated at two different temperatures.

Results: A total of 42 A. baumannii isolates were multidrug resistant. Among 21 isolates, each was ESBL and non-ESBL producers. Pellicle formation at $25^{\circ} \mathrm{C}$ in ESBL and non-ESBL producer isolates was $47.61 \%(10 / 21)$ and $28.57 \%(06 / 21)$. Pellicle formation at $37^{\circ} \mathrm{C}$ in ESBL and non-ESBL producer isolates was 57.14\% (12/21) and 42.85\% (09/21), respectively. Gelatinase production was present in 38.09\% ESBL and 28.57\% in non-ESBL producers. ESBL strains were more virulent compared to non-ESBL producers among patients of DFIs.

Conclusion: This study showed that pellicle formation at $37^{\circ} \mathrm{C}$ was highly virulent due to ESBL producers. Gelatinase production was elevated in ESBL compared to non-ESBL producer isolates. This attribute of the isolates could render ESBL positive more pathogenic. Colistin and polymyxin B are the only choices of treatment for multidrug-resistant Acinetobacter baumannii infections.

Keywords: Acinetobacter baumannii, Diabetic foot ulcer, Gelatinase, Multidrug resistance, Pellicle.

(C) 2018 The Authors. Published by Innovare Academic Sciences Pvt Ltd. This is an open access article under the CC BY license (http://creativecommons. org/licenses/by/4. 0/) DOI: http://dx.doi.org/10.22159/ajpcr.2018.v11i9.28336

\section{INTRODUCTION}

Acinetobacter baumannii is a Gram-negative Coccobacillus that causes nosocomial infections such as urinary tract infections, respiratory infections, bacteremia, secondary meningitis, and wound infections in immunodeficiency individuals including diabetic patients [1].

Among diabetic foot ulcer infection (DFI) and $14.28 \%$ A. baumannii were found symptomatic with necrotizing fasciitis, osteomyelitis, pus discharge, leukocytosis, and low immune, so it can be considered as pathogen not to be colonizer due to the threatening of limb amputation [2,3]. A. baumannii is the second most commonly found pathogen among non-fermented bacteria in DFIs. Peripheral arterial diseases are often present in patients of DFIs and may lead to the poor penetration of antibiotics into the lowerlimb tissues, and thereby, this promotes grown of resistant bacteria [4]. Few virulence factors of $A$. baumannii have been recognized; these are biofilm formation, siderophore production, cell surface hydrophobicity, gelatinase production, and pellicle formation. Gelatinase is a protease enzyme produced by bacteria in ulcers/wound lead to break down the collagen in subcutaneous tissue, casein, hemoglobin, and other bioactive peptides and also are involved in inflammation, and contribute to virulence [5]. Pellicle formation in A. baumannii allows them to survive on less or nutrient-limited surfaces for several days [6]. The pellicle formation needs different proteins that are associated with pili formation such as chaperone-usher system pili and the putative type III pili [7]. The matrix of $A$. baumannii pellicles is complex, containing different extracellular polymeric substances, that is lipopolysaccharide, exopolysaccharides, and cell surface appendages that may be contributing factors for the occurrence and prevalence of $A$. baumannii in the clinical environment which exhibits different cell properties in wound/ ulcers [8]. In the hospital ward environment, pellicles could be formed in small droplets, and therefore, may involve in bacterial colonization to pathogenic for immunodeficiency or diabetic foot ulcer patients. Bacterial bunch may eventually be detached from the pellicle and move freely to cause infection [9]. Pellicle formation is more readily formed at $25^{\circ} \mathrm{C}$ and $37^{\circ} \mathrm{C}$. Bacteria in the pellicle could revert to the abiotic surface and cause infection. Due to this reason, hospital ward disinfectants and antiseptics should target this biofilm structure and protect bacteria from external hazards [6]. Such variations and differences could pose a challenge in the development of strategies that counter biofilm formation as a convenient and general therapeutic target to treat $A$. baumannii infections in patients of diabetic foot ulcer. This study was conducted to understand the virulence factors of the ESBL, non-ESBL producers, pellicle formation, and gelatinase production by A. baumannii in DFI patients, which may lead to the development of secondary infections and non-healing ulcer and lead to amputation of lower limb extremities. The purpose of the study was to assess and compare the gelatinase activity and pellicle formation in ESBL and non-ESBL producing A. baumannii isolates from DFI.

\section{METHODS}

A prospective 1-year study was carried out in the Department of General Surgery, Microbiology, and Yenepoya Research Center, Yenepoya Medical College, Mangalore, India. A total of 42 non-duplicate ESBL and non-ESBL 
producing A. baumannii isolates were collected from September 2016 to September 2017 after obtaining Ethical Clearance from Institutional Ethics Committee (Reg.No.- YU172/2016). A. baumannii was identified using standard methods based on Gram staining, cultured on 5\% sheep blood, and MacConkey agar media, and identification was done using the BD Phoenix 100 system [10]. Antimicrobial susceptibility testing of A. baumannii was done by the Kirby-Bauer disk diffusion method in accordance with the CLSI guidelines 2016 [11]

\section{Gelatinase assay}

The isolate was grown in brain heart infusion broth and incubated at $37^{\circ} \mathrm{C}$ for $18 \mathrm{~h}$. One loopful of culture was inoculated on to Luria Bertani Agar containing gelatin $(30 \mathrm{~g} / \mathrm{L})$. The plates were incubated overnight at $37^{\circ} \mathrm{C}$ and then cooled for $5 \mathrm{~h}$ at $4^{\circ} \mathrm{C}$. The positive result was indicated by the appearance of a turbid halo around colonies on the medium for gelatinase production [5].

\section{Pellicle or air-liquid interface biofilm (ALI) assay}

Pellicle assay or ALI assay was performed by inoculating $5 \mathrm{ml}$ of Mueller-Hinton (MH) broth taken two in polystyrene tubes with a single colony of isolates of A. baumannii with an initial OD 600 to 0.01 . The tubes were then incubated for 3 days at $25^{\circ} \mathrm{C}$ or $37^{\circ} \mathrm{C}$ without shaking. Pellicle formation if any; appeared as a white layer at the surface of $\mathrm{MH}$ broth [7]. The negative control was used by A. baumannii ATCC 19606, and positive control of Pseudomonas aeruginosa (PA01) was maintained simultaneously.

\section{Statistical analysis}

Statistical analysis was performed using SPSS 23.0 version software (SPSS Inc., Chicago, IL). Descriptive statistics like frequency (\%) were used for analysis. Variables were expressed as percentages. All the data were expressed as table diagrams.

\section{RESULTS}

A total of 42 isolates of A. baumannii; 21 each from ESBL and nonESBL producers from DFI were assessed in the study. All the patients were typed 2 diabetes mellitus and comorbidities with hypertension, nephropathy, neuropathy, retinopathy, and peripheral vascular diseases. The age group of these patients ranged from 30 to 80 years, and the maximum number of patients was in the age group of 44 to 64 years. Male mean age $(55.55 \pm 10.25)$ and female mean age $(52.56 \pm 10.38)$ were male predominant compared to female.

\section{Gelatinase assay}

All the isolates were multidrug resistant among ESBL and non-ESBL producer in A. baumannii ( $\mathrm{n}=21$ each), positive gelatinase producers were observed 8/21(38.09\%) and 6/21 (28.57\%), respectively (Fig. 1 and Table 1). Gelatinase production was absent in 13/21 (61.90\%) of ESBL and 15/21 (71.43\%) of non-ESBL producers. ESBL producers were more gelatinase producers compared to non-ESBL producers.

\section{Pellicle formation or ALI assay}

After $24 \mathrm{~h}$ of incubation; a thin pellicle began to form at the surface of the liquid media; which further grew in thickness and by the end of the third day; an opaque and solid structure covered the entire liquid surface. $42 \mathrm{~A}$. baumannii from diabetic foot ulcer isolates was visually analyzed for pellicle formation, and 16 (38.09\%) isolates tested positive. Among pellicle forming isolates, ESBL and non-ESBL were $10 / 21$ (47.61\%) and 6/21 (28.57\%), respectively (Fig. 2 and Table 2).

\section{DISCUSSION}

Nearly $15-25 \%$ of type 2 diabetes mellitus patients experience DFI during their lifetime. The predominance is more in males compared to female patients [12]. DFIs occur mainly due to polymicrobial and monomicrobial infections [13]. Multidrug resistance and production of ESBL by microbes and their infections are associated with the increased length of hospital stay, with higher morbidity, mortality rate, and cost burden to the patients. The virulent factors were more in ESBL isolates compared to non-ESBL isolates. A. baumannii, considered to be a low-

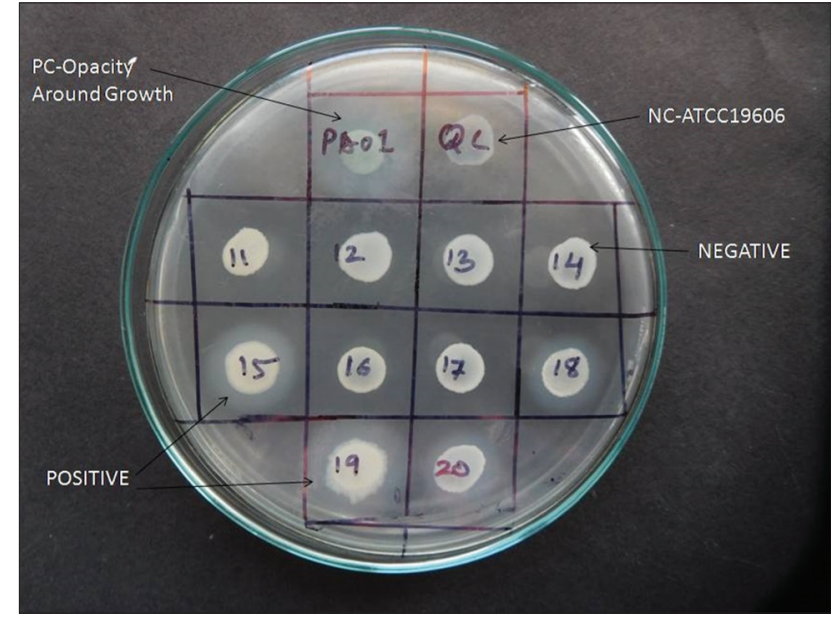

Fig. 1: Gelatinase production in Acinetobacter baumannii from extended-spectrum beta-lactamase producers isolates. DFI: Diabetic foot ulcer infection; NC: Negative control (A. baumannii ATCC19606);

PC: Positive control (PA01=Pseudomonas aeruginosa 01)

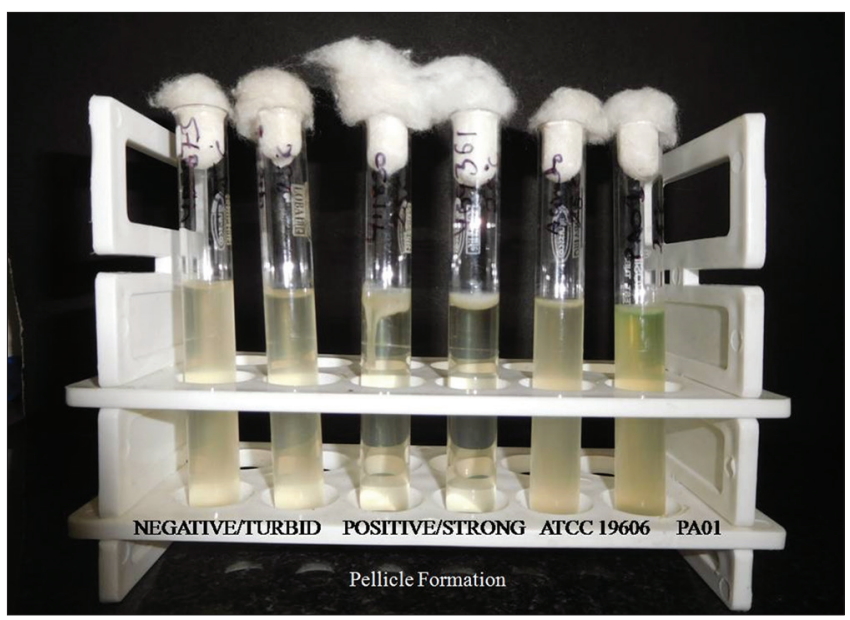

Fig. 2: Pellicle formation or air-liquid interface biofilm in Acinetobacter baumannii from Extended-spectrum betalactamase producers isolates. PA01: Pseudomonas aeruginosa 01

Table 1: Association of A. baumannii in ESBL isolates among pellicle formation and gelatinase production from $D F I$

\begin{tabular}{lll}
\hline $\begin{array}{l}\text { Acinetobacter baumannii } \\
\text { ESBL (n=21) }\end{array}$ & Present (\%) & Absent (\%) \\
\hline $\begin{array}{l}\text { Pellicle or ALI at } 25^{\circ} \mathrm{C} \text { for } 3 \text { days } \\
\text { Pellicle or ALI at } 37^{\circ} \mathrm{C} \text { for } 3 \text { days }\end{array}$ & $10(47.61)$ & $11(52.38)$ \\
$\begin{array}{l}\text { Both } 25^{\circ} \mathrm{C} \text { and } 37^{\circ} \mathrm{C} \text { for } 3 \text { days } \\
\text { incubation }\end{array}$ & $10(47.61)$ & $9(42.86)$ \\
Gelatinase production & $8(38.09)$ \\
\hline
\end{tabular}

ESBL: Extended-spectrum beta-lactamase, ALI: Air-liquid interface biofilm, DFI: Diabetic foot ulcer infection

grade pathogen indeed has many putative virulence factors. It causes a significant major problem in immunodeficiency or diabetic patients.

Our study showed gelatinase production by 38.09\% ESBL and 28.57\% non-ESBL isolates of A. baumannii. Surprisingly, none of the earlier studies have reported ESBL and non-ESBL types in DFI isolates of $A$. baumannii. Some studies reported gelatinase production in A. baumannii, but it is not clear whether specimens/sample were taken from the patient with DFI or not. Hence, this study is the first report ESBL and non-ESBL production in A. baumannii from DFI patients. 
Table 2: Association of A. baumannii in non-ESBL isolates among pellicle formation and gelatinase production from DFI

\begin{tabular}{lll}
\hline Non-ESBL (n=21) & Present (\%) & Absent (5) \\
\hline $\begin{array}{l}\text { Pellicle or ALI at } 25^{\circ} \mathrm{C} \text { for 3 days } \\
\text { Pellicle or ALI at } 37^{\circ} \mathrm{C} \text { for 3 days }\end{array}$ & $6(28.57)$ & $15(71.43)$ \\
$\begin{array}{l}\text { Both } 25^{\circ} \mathrm{C} \text { and } 37^{\circ} \mathrm{C} \text { for } 3 \text { days } \\
\text { incubation }\end{array}$ & $4(19.05)$ & $12(57.14)$ \\
Gelatinase production & $6(28.57)$ & $9(42.86)$ \\
\hline
\end{tabular}

ALI: Air-liquid interface biofilm, ESBL: Extended-spectrum beta-lactamase,

DFI: Diabetic foot ulcer infection

Cevahir et al. [14] reported that gelatinase activity occurred in 14\% A. baumannii infections. Rasha et al. [15] reported $100 \%$ gelatinase production by A. baumannii isolates.

Pellicle formation assay in our study showed that among 42 A. baumannii from diabetic foot ulcer isolates; $38.09 \%$ pellicle forming isolates were found. Pellicle formation at $25^{\circ} \mathrm{C}$ and $37^{\circ} \mathrm{C}$ in ESBL and non-ESBL each was $47.61 \%$ and $28.57 \% ; 57.14 \%$ and $42.85 \%$, respectively. Similarly, Marti et al. [7] reported that pellicle formation at $25^{\circ} \mathrm{C}$ and $37^{\circ} \mathrm{C}$ was $35.9 \%$ and $12.2 \%$, respectively. Another study by Chabane et al. [6] reported pellicle formation or air-liquid interface biofilm formation at $25^{\circ} \mathrm{C}$ at $30.32 \%$ in A. baumannii isolates. Sara et al. [16] reported that the A. baumannii group has a higher ability to form pellicle than other than $A$. baumannii and this feature could be connected to the higher pathogenic rate of A. baumannii and probably is contributing factor to the increased risk of clinical infection. In our study, colistin and polymyxin B only effective drug for multidrug-resistant isolates of diabetic foot ulcer patients. Similarly, Sadia et al. [17] reported, sensitive was $98 \%$ colistin and polymyxin B to stop the rapid spreading of ESBL producers. A. baumannii has a higher ability to form a pellicle or air-liquid interface biofilm than other species, it could be connected to the higher colonization rate of patients by pathogenic A. baumannii, and probably contribute to the increased risk of DFI. The Gelatinase is a proteolytic enzyme that is capable of hydrolyzing gelatin, which can traverse the cell membrane and hydrolyze collagen in subcutaneous tissues during wound infections. It's linked to inflammation, therefore, to contribute to the virulence on a human.

\section{CONCLUSION}

Pellicle formation or air-liquid interface biofilm formation and gelatinase production by $A$. baumannii was high in ESBL producers compared to non-ESBL producer isolate from DFIs. Virulence factors play a significant role in multidrug-resistant $A$. baumannii infections. On that point numerous factors were studied in which the pellicle contribution was maximized to the infection from diabetic foot ulcers in patients. Understanding, these elements that influence the virulence of this organism will help in recognition of these pathogenic isolates with high or low potential virulence. Colistin and polymyxin $\mathrm{B}$ are the only choices of the drug against multidrugresistant $A$. baumannii infection.

\section{ACKNOWLEDGMENT}

We would like to thank Yenepoya Research Center, Department of General Surgery and Microbiology, for supporting this study and Yenepoya (Deemed to be University), for providing a research grant for this study.

\section{AUTHOR'S CONTRIBUTION}

Diwan Mahmood Khan has contributed to the study conception, design, sample collection, analysis and interpretation of the data, and manuscript preparation. Dr. I. Venkatakrishna Rao and Dr. M. S. Moosabba have contributed to the study conception and critical revision of the manuscript. All the authors have contributed equally to the communication of the study, writing, and editing the article.

\section{CONFLICTS OF INTEREST}

None of the authors have any conflicts of interest to be declared

\section{REFERENCES}

1. Bergogne-Bérézin E, Towner KJ. Acinetobacter spp. As nosocomial pathogens: Microbiological, clinical, and epidemiological features. Clin Microbiol Rev 1996;9:148-65.

2. Lahiri KK, Mani NS, Purai SS. Acinetobacter spp as nosocomial pathogen: Clinical significance and antimicrobial sensitivity. Med J Armed Forces India 2004;60:7-10.

3. Murali TS, Kavitha S, Spoorthi J, Bhat DV, Prasad AS, Upton Z, et al. Characteristics of microbial drug resistance and its correlates in chronic diabetic foot ulcer infections. J Med Microbiol 2014;63:1377-85.

4. Vatan A, Saltoglu N, Yemisen M, Balkan II, Surme S, Demiray T, et al. Association between biofilm and multi/extensive drug resistance in diabetic foot infection. Int J Clin Pract 2018:e13060.

5. Franco G, Costa DM, Cristina M, Tognim B, Cardoso CL, CarraraMarrone FE, et al. Preliminary evaluation of adherence on abiotic and cellular surfaces of Acinetobacter baumannii strains isolated from catheter tips. Braz J Infect Dis 2006;10:346-51.

6. Nait Chabane Y, Marti S, Rihouey C, Alexandre S, Hardouin J, Lesouhaitier O, et al. Characterisation of pellicles formed by Acinetobacter baumannii at the air-liquid interface. PLoS One 2014;9:e111660.

7. Martí S, Rodríguez-Bão J, Catel-Ferreira M, Jouenne T, Vila J, Seifert $\mathrm{H}$, et al. Biofilm formation at the solid-liquid and air-liquid interfaces by Acinetobacter species. BMC Res Notes 2011;4:2-5.

8. Peleg AY, Seifert H, Paterson DL. Acinetobacter baumannii: Emergence of a successful pathogen. Clin Microbiol Rev 2008;21:538-82.

9. McConnell MJ, Actis L, Pachón J. Acinetobacter baumannii: Human infections, factors contributing to pathogenesis and animal models. FEMS Microbiol Rev 2013;37:130-55.

10. Endimiani A, Luzzaro F, Tamborini A, Lombardi G, Elia V, Belloni R, et al. Identification and antimicrobial susceptibility testing of clinical isolates of nonfermenting gram-negative bacteria by the phoenix automated microbiology system. New Microbiol 2002;25:323-9.

11. Clinical and Laboratory Standards Institute (CLSI). Performance Standards for Antimicrobial Susceptibility Testing. 26 ${ }^{\text {th }}$ ed Wayne PA: CLSI Supplements M100S; 2016.

12. Kumar SL, Ashutosh SR, Gokulshankar S, Ranjith MS, Mohanty BK, Lim MY. Is bacteriology a contributing factor in unsalvageable nature of diabetic foot infections? A study in a district hospital in Malaysia. Int J Pharm Pharm Sci 2015;8:262-5.

13. Kateel R, Augustine AJ, Prabhu S, Ullal S, Pai M, Adhikari P. Clinical and microbiological profile of diabetic foot ulcer patients in a tertiary care hospital. Diabetes Metab Syndr Clin Res Rev 2018;12:27-30.

14. Cevahir N, Demir M, Kaleli I, Gurbuz M, Tikvesli S. Evaluation of biofilm production, gelatinase activity, and mannose-resistant hemagglutination in Acinetobacter baumannii strains. J Microbiol Immunol Infect 2008;41:513-8

15. Rasha JM, Al-Warid AL, Al-Thahab IJ. Isolation and identification of Acinetobacter baumannii in Hilla city. Int J Adv Biol Res 2014;4:4-8.

16. Giles SK, Stroeher UH, Eijkelkamp BA, Brown MH. Identification of genes essential for pellicle formation in Acinetobacter baumannii. BMC Microbiol 2015;15:116.

17. Zafar S, Naqvi SB, Abbas T, Qazi1 F. Synergistic combinations of broad-spectrum antibiotics against Acinetobacter spp. Int J Pharm Pharm Sci 2015;7:214-7 